\title{
A Simple Procedure to Determine Complex Permittivity of Moist Materials Using Standard Commercial Coaxial Sensor
}

\author{
M. A. Jusoh, Z. Abbas*, J. Hassan, B. Z. Azmi, and A. F. Ahmad \\ Department of Physics, Faculty of Science, Universiti Putra Malaysia, 43400 Serdang Selangor, Malaysia, \\ *za@science.upm.edu.my
}

\begin{abstract}
A simple procedure was developed to determine complex permittivity of moist materials for known percentage of moisture content at any frequency based using a standard commercial coaxial sensor. Polynomial fitting and Gaussian elimination method were applied to obtain a single equation of complex permittivity as a function of frequency and moisture content. The empirical equation was tested for new samples and was found to have mean error percentage of $5.14 \%$ and $10.22 \%$ for dielectric constant and loss factor, respectively, when compared to a commercial probe.
\end{abstract}

Keywords: Open ended coaxial line, permittivity, maize, empirical model

\section{INTRODUCTION}

$\mathrm{A}$ N OPEN ENDED coaxial line has been used by many researchers for measuring the complex permittivity of materials nondestructively [1]-[4]. In this method, the sample is placed against an open end of a coaxial line and its reflection coefficient is measured. A coaxial line having inner and outer radii aand $b$, respectively, filled with a lossless homogeneous dielectric having a relative permittivity is terminated in the plane $\mathrm{z}=0$ onto a flat metallic flange extending theoretically to infinity in the transverse direction [1]. The material terminating the aperture is assumed to be homogeneous, isotropic, linear, and nonmagnetic, of complex permittivity extending to infinity. The schematic diagram of an open ended coaxial sensor with a sample is shown in Figs.1a and $1 \mathrm{~b}$.

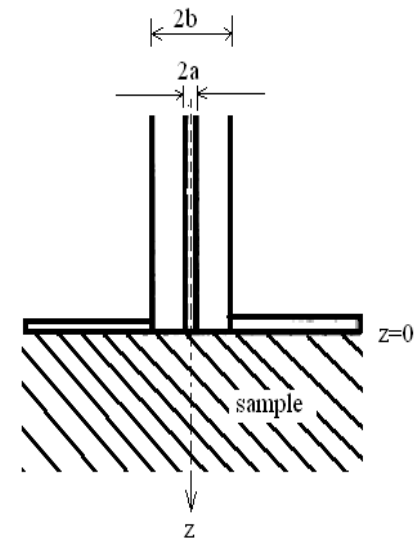

(a)

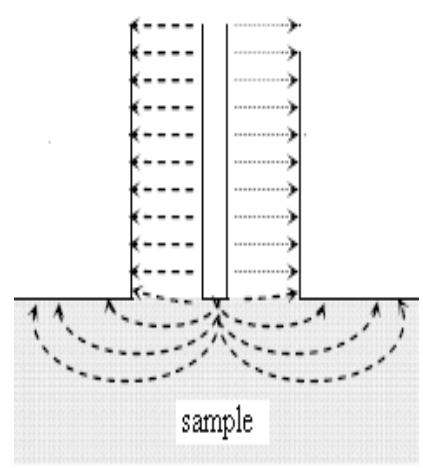

(b)
Fig.1. Open ended coaxial sensor [6] (a) Schematic diagram (b) Electric field distribution.

\section{SUBJECT \& METHODS}

The most commonly used theoretical model for calculating the complex permittivity is the dielectric mixture model. The dielectric mixture is described in terms of the fractional volume and permittivity of each constituent. There are several mixture models that have been proposed [5].
There are Kraszewski (Kraszewski equation)

$$
\sqrt{\varepsilon^{*}}=v_{1} \sqrt{\varepsilon_{1}}+v_{2} \sqrt{\varepsilon_{2}}+v_{3} \sqrt{\varepsilon_{3}}
$$

Landau, Lifshitz and Looyenga, (Landau equation)

$$
\sqrt[3]{\varepsilon^{*}}=v_{1} \sqrt[3]{\varepsilon_{1}}+v_{2} \sqrt[3]{\varepsilon_{2}}+v_{3} \sqrt[3]{\varepsilon_{3}}
$$

Lichtenecker, (Lichtenecker equation)

$$
\ln \varepsilon^{*}=v_{1} \ln \varepsilon_{1}+v_{2} \ln \varepsilon_{2}+v_{3} \ln \varepsilon_{3}
$$

The notation used here applies to three component mixtures where $\varepsilon^{*}$ represent the complex permittivity of the mixture, $\varepsilon_{1}$ is the permittivity of medium $1, \varepsilon_{2}$ is the permittivity of medium 2 and $\varepsilon_{3}$ is the permittivity of medium 3. The $v_{1}, v_{2}$ and $v_{3}$ are the fractional volume of the respective components, where $v_{1}+v_{2}+v_{3}=1$ as the maize mixture consist of three main components. There are volume fractions of water, oil and fibre and this can be expressed as

$$
v_{\text {water }}+v_{\text {fibre }}+v_{\text {oil }}=1
$$

The permittivity of each component was measured using Agilent $85070 \mathrm{~B}$ as listed in Table 1. The volume fraction of fibre is 0.17 [6].

The determination of the complex permittivity of maize begins with the establishment of an empirical model for moisture content $(\mathrm{mc})$ in maize kernel. Typically, the diameter of a maize kernel is between 8 and $12 \mathrm{~mm}$. The measurements were carried out at room temperature, $25^{\circ} \mathrm{C}$ for 250 kernels of maize with variation of moisture content from the youngest to the oldest fruit. It has been found that the moisture content for the youngest and the oldest kernel is $80 \%$ and $10 \%$, respectively. The maize kernels were dried 
at $103 \pm 1{ }^{\circ} \mathrm{C}$ up to 72 hours using the air oven technique in accordance with the American Association of Cereal Chemist (AACC) techniques 44-15A [7].

Table 1. Dielectric constant and loss factor of water, oil and fibre at different frequencies

\begin{tabular}{|c|c|c|c|}
\hline $\begin{array}{c}\text { Freq } \\
(\mathrm{GHz})\end{array}$ & $\varepsilon_{1}$ (water) & $\varepsilon_{2}$ (oil) & $\varepsilon_{3}$ (fibre) \\
\hline 1 & $78.2804-3.7957 \mathrm{i}$ & $2.8977-0.1852 \mathrm{i}$ & $3.9915-0.6372 \mathrm{i}$ \\
\hline 2 & $77.6916-7.5552 \mathrm{i}$ & $2.8193-0.1784 \mathrm{i}$ & $3.7943-0.5728 \mathrm{i}$ \\
\hline 3 & $76.7273-11.1869 \mathrm{i}$ & $2.784-0.192 \mathrm{i}$ & $3.5902-0.5785 \mathrm{i}$ \\
\hline 4 & $75.4209-14.6423 \mathrm{i}$ & $2.7739-0.1969 \mathrm{i}$ & $3.5247-0.6014 \mathrm{i}$ \\
\hline 5 & $73.8031-17.9119 \mathrm{i}$ & $2.7489-0.24 \mathrm{i}$ & $3.4024-0.5588 \mathrm{i}$ \\
\hline
\end{tabular}

The commercial sensor configuration and the calculation of permittivity were initially calculated using the procedure given in [8] for the frequency ranges $1 \mathrm{GHz}$ to $5 \mathrm{GHz}$ at room temperature. It has been found that the dielectric constant and loss factor changes with frequency in a linear form and the $4^{\text {th }}$ order polynomial form, respectively, as shown in Figs. 2 and 3. Relationship between the dielectric constant and loss factor with frequency can be described as

and

$$
\varepsilon^{\prime}(\mathrm{mc}, f)=\mathrm{A}(\mathrm{mc}) f+\mathrm{B}(\mathrm{mc})
$$

$$
\varepsilon^{\prime \prime}(\mathrm{mc}, f)=\mathrm{C}(\mathrm{mc}) f^{4}+\mathrm{D}(\mathrm{mc}) f^{3}+\mathrm{E}(\mathrm{mc}) f^{2}+\mathrm{F}(\mathrm{mc}) f+\mathrm{G}(\mathrm{mc})
$$

where, $A, B, C, D, E, F$, and $G$ are unknown variables that will be determined.

\section{RESULTS AND DISCUSSION}

The relationship between both dielectric constants, $\varepsilon^{\prime}$ and loss factor, $\varepsilon^{\prime \prime}$ of maize from $1 \mathrm{GHz}$ to $5 \mathrm{GHz}$ at various percentages of moisture content is shown in Figs.2a and $2 \mathrm{~b}$. It has been observed that the dielectric constant and loss factor are almost constant at low moisture content except for high moisture content. It is due to the small dispersion at low moisture content. The loss factor, $\varepsilon^{\prime \prime}$, decreases at low frequency, but increases again at $2 \mathrm{GHz}$. This behavior is influenced by ionic conductivity at lower frequencies, around $2 \mathrm{GHz}$, since the dielectric properties in agricultural products are primarily influenced by their ionic conductivity of fluids contained in their cellular structure [2]. The dielectric losses decrease with frequency according to $\sigma \omega^{-1} \varepsilon_{0}^{-1}$ [9] due to ionic conductivity. At low moisture content, it is difficult to measure moisture content since the moisture content in a maize kernel is non-uniform. That is why the increasing is not uniform at moisture content below $30 \%$, whereby there is only a small difference between $17 \%$ and $22 \%$ moisture content, but the difference is much greater between $22 \%$ and $24 \%$ moisture content. This is probably due to the lack of accuracy of the Agilent coaxial probe sensor for reflection measurement of samples having low moisture, since the moisture content in a maize kernel is non-uniform. This is usually attributted to the effect of bound water detailed in [10]. Thus, this work only considers moisture content range between $32 \%$ and $80 \%$ moisture content.

Noisy behavior that occurs in 56\% and 59\% moisture content as shown in Fig.2 is probably caused by measurement errors during experimental work.

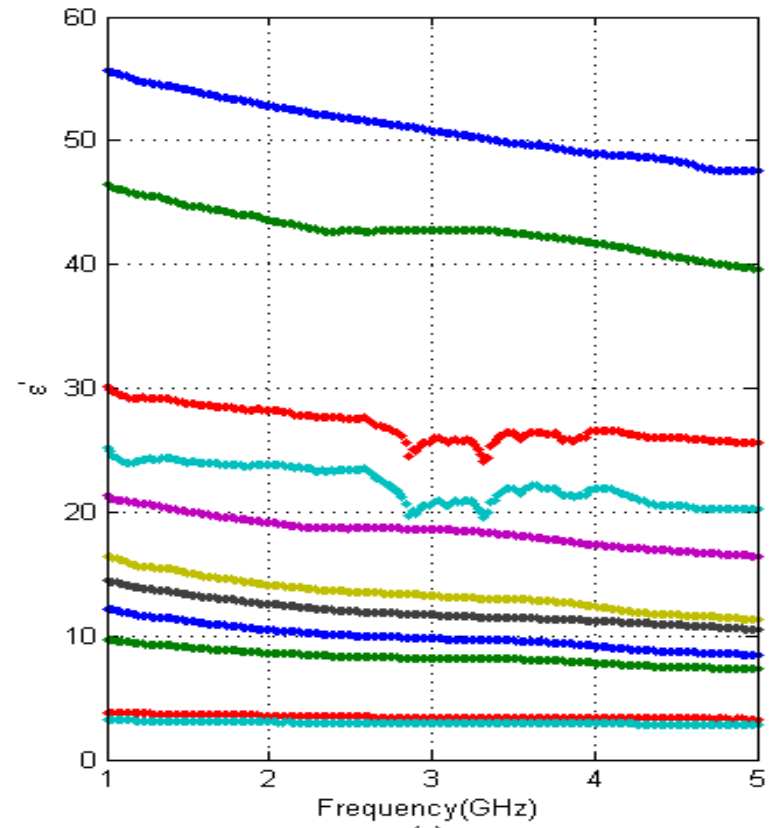

(a)

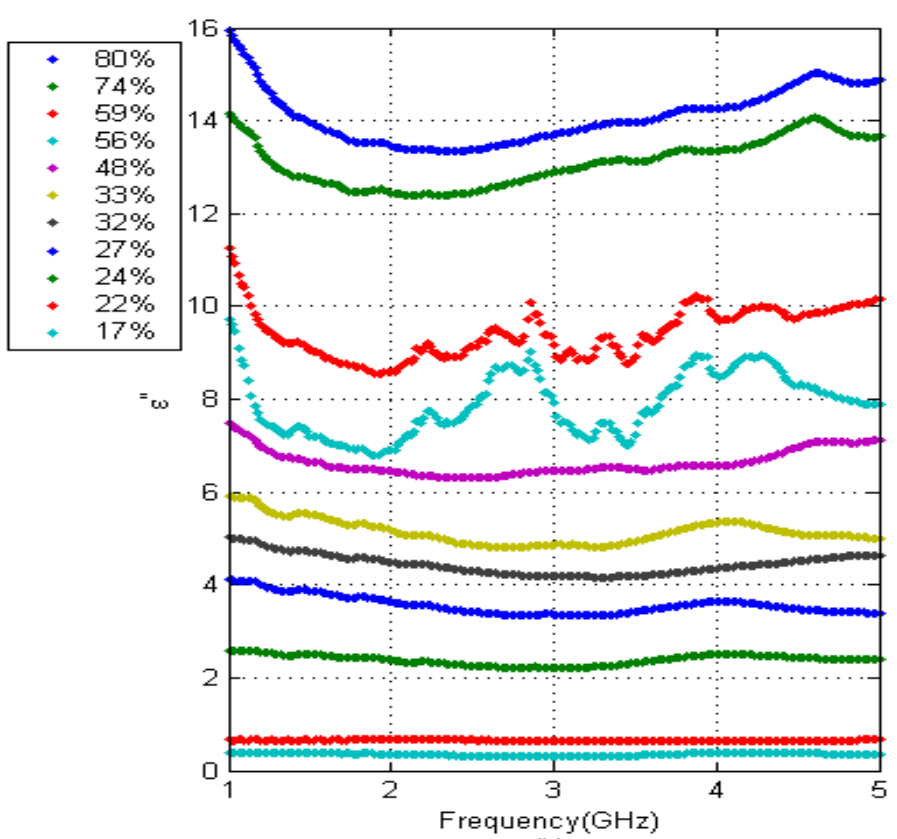

(b)

Fig.2. Relationship between (a) dielectric constant and (b) loss factor at various moisture contents for a maize kernel. 
Table 2. Unknown variable as a function of moisture content for real and imaginary part of complex permittivity

\begin{tabular}{|c|c|}
\hline$\varepsilon^{*}$ & $(A, B, C, D, E, F$ and $G)$ \\
\hline$\varepsilon^{\prime}$ & $\begin{array}{l}A=9.0913 \times 10^{-7} m c^{4}-1.9672 \times 10^{-4} m c^{3}+1.4922 \times 10^{-2} m c^{2}-0.4870 m c+4.943 \\
B=-1.4572 \times 10^{-6} m c^{4}+6.4072 \times 10^{-4} m c^{3}-6.4415 \times 10^{-2} m c^{2}+2.9131 m c-32.071\end{array}$ \\
\hline$\varepsilon^{\prime \prime}$ & $\begin{array}{l}C=1.3796 \times 10^{-7} m c^{4}-3.0113 \times 10^{-5} m c^{3}+2.2756 \times 10^{-3} m c^{2}-0.0668 m c+0.6214 \\
D=-2.2409 \times 10^{-6} m c^{4}+4.7203 \times 10^{-4} m c^{3}-3.4442 \times 10^{-2} m c^{2}+0.9797 m c-8.9526 \\
E=1.1238 \times 10^{-5} m c^{4}-2.3274 \times 10^{-3} m c^{3}+0.1668 m c^{2}-4.6510 m c+41.954 \\
F=-1.8791 \times 10^{-5} m c^{4}+3.8955 \times 10^{-3} m c^{3}-0.2791 m c^{2}+7.7051 m c-68.850 \\
G=2.0664 \times 10^{-6} m c^{4}-5.3044 \times 10^{-4} m c^{3}+4.4260 \times 10^{-2} m c^{2}-0.9674 m c+6.0431\end{array}$ \\
\hline
\end{tabular}

The unknown variables $A, B, C, D, E, F$, and $G$ were determined using the Gaussian Elimination method. The unknown variables as a function of moisture content are tabulated in Table 2. The empirical fitting for the determination of complex permittivity was established as described in Table 2. The empirical model was used to estimate the complex permittivity as a function of moisture content and was compared with measured and calculated permittivity that were found using selected mixture models such as Kraszewski, Landau and Lichtenecker.
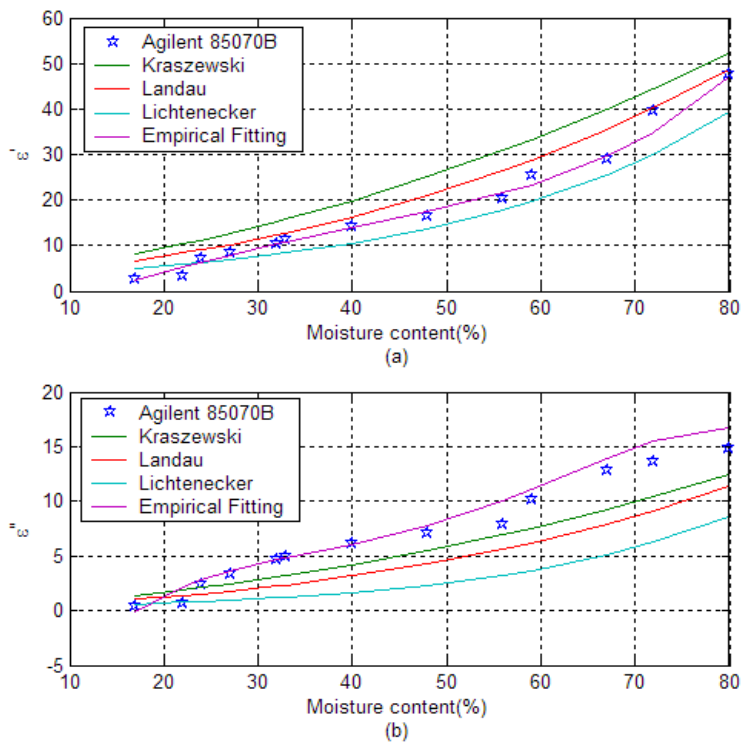

Fig. 3. Relationship between (a) dielectric constant and (b) loss factor with moisture content for experimental results, mixture models (Kraszewski, Landau and Lichtenecker) and empirical fitting at $5 \mathrm{GHz}$.

The result for the empirical model shows a good agreement with experimental results compared with the mixture model for both dielectric constant and loss factor. It is clearly shown in Fig. 3, whereby the empirical fitting is the closest to the measured data. The accuracy of the empirical model can be described using relative error. The relative error for the empirical model and mixture model is based on the measurement data as described in (7).

$$
\text { relative error }=\left|\frac{\text { Measured }-X}{\text { Measured }}\right|
$$

where the measured data was obtained from Agilent 85070B and $\mathrm{X}$ is either Kraszewski, Landau, Lichtenecker or empirical fitting. The comparisons of the relative error for empirical model, Kraszewski mixture model (1), Landau mixture model (2) and Lichtenecker mixture model (3) are shown in Tables 3 and 4 for the dielectric constant and loss factor, respectively.

Table 3. Relative error for calculated dielectric constant

\begin{tabular}{ccccc}
\hline \multicolumn{5}{c}{ Dielectric constant } \\
\hline MC(\%) & Empirical & Eq. 1 & Eq. 2 & Eq. 3 \\
\hline 32 & 0.0099 & 0.5361 & 0.2748 & 0.2545 \\
33 & 0.0383 & 0.4851 & 0.2445 & 0.2815 \\
48 & 0.0684 & 0.6236 & 0.3773 & 0.2183 \\
56 & 0.0618 & 0.6272 & 0.4108 & 0.2010 \\
59 & 0.0880 & 0.4216 & 0.2720 & 0.2724 \\
67 & 0.0164 & 0.4930 & 0.3506 & 0.2126 \\
72 & 0.1189 & 0.2898 & 0.2314 & 0.2868 \\
80 & 0.0095 & 0.2787 & 0.2397 & 0.2522 \\
\hline Mean & 0.0514 & 0.4694 & 0.3001 & 0.2474 \\
\hline
\end{tabular}

Table 4. Relative error for calculated loss factor

\begin{tabular}{ccccc}
\hline \multicolumn{5}{c}{ Loss factor } \\
\hline MC(\%) & Empirical & Eq. 1 & Eq. 2 & Eq. 3 \\
\hline 32 & 0.0035 & 0.3432 & 0.5164 & 0.7477 \\
33 & 0.0360 & 0.3615 & 0.5284 & 0.7544 \\
48 & 0.0798 & 0.2331 & 0.3992 & 0.6745 \\
56 & 0.2743 & 0.1191 & 0.2834 & 0.5884 \\
59 & 0.0875 & 0.2589 & 0.3882 & 0.6390 \\
67 & 0.0769 & 0.2832 & 0.3846 & 0.6049 \\
72 & 0.1310 & 0.2390 & 0.3301 & 0.5431 \\
80 & 0.1288 & 0.1671 & 0.2373 & 0.4208 \\
\hline Mean & 0.1022 & 0.2506 & 0.3834 & 0.6216 \\
\hline
\end{tabular}


Tables 3 and 4 show clearly that the empirical model give the smallest relative error for the dielectric constant and loss factor compared to the mixture model. The relative error for loss factor is higher compared to the dielectric constant due to the range between loss factor of water and dry maize, which is smaller compared to the dielectric constant. It means that the sensitivity for determination of the dielectric constant of maize is higher compared to loss factor.

\section{CONCLUSION}

The empirical equation of complex permittivity as described in Table 2 is a combination between real and imaginary part. This equation can be used to predict the dielectric constant and loss factor of maize. This equation is best for the prediction of moisture content between $32 \%$ and $80 \%$ with percentage mean error $5.14 \%$ and $10.22 \%$ for the dielectric constant and loss factor, respectively. This model is applicable only in the frequency range $1 \mathrm{GHz}$ to $5 \mathrm{GHz}$.

\section{ACKNOWLEDGEMENT}

This research is funded by the Ministry of Science and Technology under the Research University Scheme (Project code: 05-01-07-0233RU)

\section{REFERENCES}

[1] Poumaropoulos, C., Misra, D. (1993). A study on the coaxial aperture electromagnetic sensor and its application in material characterization. In IEEE Instrumentation and Measurement Technology Conference, 18-20 May 1993, 52-55.
[2] Mudgett, R.E., Goldblith, S.A., Wang, D.I.C., Westphal, W.B. (1977). Prediction of dielectric properties in solid foods of high moisture content at ultrahigh and microwave frequencies. J. Food. Proc. Pres., 1 (2), 119-151.

[3] Weiner, M.M., Cruze, S.P., Li, C.C., Wilson, W.J. (1987). Monopole Elements on Circular Ground Plane. New York: Artech House.

[4] Abbas, Z., You, K.Y., Shaari, A.H., Zakaria, A., Hassan, J. (2005). Fast and accurate technique for determination of moisture content in oil palm fruits using open-ended coaxial sensor. Jap. J. Appl. Phys., 44, 5272-5274.

[5] Subedi, P., Chatterjee, I. (1993). Dielectric mixture model for asphalt-aggregate mixtures. J. Microwave Power Electromagn., 28 (2), 68-72.

[6] Fakirov, S., Bhattacharyya, D. (2007). Handbook of Engineering Biopolymers: Homopolymers, Blends and Composites. Hanser Verlag.

[7] Multon, J.L. (1996). Quality Control for Foods and Agricultural Products. Wiley.

[8] Blackham, D.V., Pollard, R.D. (1997). An improved technique for permittivity measurements using a coaxial probe. IEEE Trans. Instrum. Meas., 46 (5), 1093-1099.

[9] Kelleners, T.J., Robinson, D.A., Shouse, P.J., Ayars, J.E., Skaggs, T.H. (2005). Frequency dependence of the complex permittivity and its impact on dielectric sensor calibration in soils. Soil Sci. Soc. Am. J., 69, 67-76.

[10] Binner, J.G.P. (ed.) (1992). Microwave Processing of Materials. Woodhead Publishing.

Received November 2, 2010. Accepted March 22, 2011. 